Keywords: cardiac tamponade; right atrial rupture; sternal fracture. Prehosp Disast Med 2002;17:s26-27.

\section{The Effects on the Central Nervous System Induced by Tetrodotoxin Poisoning}

Francis Fu Sheng Wui ${ }^{1}$ Chi-Wen Juan $;{ }^{1}$ Pang-Cbi Lai $;{ }^{2}$

Kuo-Chou Cheng; ${ }^{1}$ Chia-Ju $\mathrm{Wu} ;{ }^{2} \mathrm{Hsiu}_{\text {-Hui Hou }}{ }^{2}$

1. Department of Emergency Medicine, Changhua Christian Hospital, Changua, Taiwan, ROC

2. Department of Nursing, Changhua Christian Hospital, Changua, Taiwan, ROC

Objective: Tetrodotoxin (TTX) is a neurotoxin known to cause food poisoning. It is found in a variety of both freshwater and marine species. Patients with tetrodotoxin intoxication generally have a very typical course of illness involving the cardiovascular, respiratory, peripheral and central nervous systems.

Methods: A 59-year-old, male, taxi driver presented to the emergency department with dyspnea and numbness after eating some porridge cooked with Takifugu niphobles. On arrival, he was tachypneic, hypertensive, consciousness clear, and was able to give a coherent history. He immediately was intubated, but respiratory effort ceased several minutes later. About one hour later, he was in deep coma with Glasgow Coma Scale (GCS) Score $=3$, had a sinus bradycardia, and became severely hypotensive.

Results: During the next two days, he continued to have fluctuating blood pressure, hypothermia, and polyuria. At 36 hours after admission, he regained consciousness and some upper limb motor function, but still had no spontaneous breathing effort. An EEG showed "minor diffuse cortical dysfunction likely to be metabolic in origin". Later in the day his vision recovered spontaneously. Ventilatory function returned and the patient self-extubated on the following day. On day 10, he was discharged after a follow-up EEG that showed "no evidence of focal cortical dysfunction". Tetrodotoxin measured by bioassay was 300 mouse units per gram (MU/g).

Conclusion: To our knowledge this is the first report of a human that demonstrated this cortical dysfunction on EEG during tetrodotoxin poisoning.

Keywords: central nervous system; intoxication; takifugu niphobles; tetrodotoxin

Prehosp Disast Med 2002;117:s27.

\section{Six Years Experience with Telemedicine in an \\ Emergency Department in Taiwan}

Lee-Min Wang, $M D ;^{1}$ Hong-Chang Lo, $M D{ }^{1}$

Chen-Hsen Lee, MD,; Dab-Dian Tang; ${ }^{2}$ Pi-Chin Yu, ${ }^{3}$

Low-Tone Ho, $M D^{3}$

1. Emergency Department

2. Information Services Center

3. Department of Medical Research and Education, Veterans General Hospital-Taipei; National Defense Medical Center.National Yang-Ming University. Taipei, Taiwan ROC

Objective: To observe telemedicine in an emergency department between Taipei-Veterans General Hospital and Kinmen-Granite, County Hospitals in Taiwan.

Methods: This clinical study was carried out from July 1996 through to September 2001. Data for total of 920 teleconsultation cases were collected using a questionnaire. Evaluation elements included: 1) consulting quality; 2) duration of the teleconsultation; the benefit to the patients; and 4) the charges. The purposes of the teleconsultation project were to establish electronically an exchange of medical information and to conduct clinical examinations and consultations.

Results: The peak interval of the ages of the patients was 21-30 years. Radiological consultation was the most frequently involved area of consultation, followed by chest medicine, neurosurgery, orthopedics, dermatology, and general surgery. The questionnaire indicated that $74 \%$ of the time, teleconsultations was used to obtain a the second opinion. More than $95 \%$ of the physicians involved believe that the telemedicine system will affect the quality of the consultation and that the consultation is helpful. The doctors in Taipei-VGH were more satisfied with the teleconsultation facility than were the doctors in Kinmen Hospitals.

Conclusion: The consultation doctors confirm that the clinical evaluation using the telemedicine is positive and has the potential to help during a disaster period.

Keywords: consultation; questionnaire; telecommunication, telemedicine.

Prebosp Disast Med 2002;17:s27.

\section{Covering Disasters: A Look at Disaster News Reporting in the Philippine Media \\ Lirio Racho Luyun \\ Cemshad, Inc., The Philippines}

Objectives: The study aims to find out how disaster news are framed in the Philippine print media and the media practices that inform the framing of such news.

Method: The study qualitatively content analyzed several disaster news reports from different broadsheets within 2001.

Results: The regularity of events that result in disasters that strike the Philippines for the past years reveals a pattern that the press applies in framing disaster news. Emphasis is given to statistics regarding the number of persons affected by these events. It also showed a tendency to place emphasis on personalities, spending precious ink to highlight the activities of politicians and officials who often use such disasters as photo opportunities to further their political careers. Disaster news, on the whole, is presented as an 'event', given prominence for a while until a new event takes its place in the public spotlight.

Conclusions: The tendency of the press to treat disaster news as 'events' has the potential to instill a feeling of fatigue among the populace towards disasters. Instead of communicating information that will help people make critical and informed choices during such disasters, the press focus on simple cause and effect explanations as to why such disasters happen. This has not translated into 